\title{
Normal bystander keratinocytes protected by oleic acid against low dose radiation induced factor's
}

\begin{abstract}
To investigate the protective role of Oleic acid against the cytotoxic mediators present in the low dose irradiated cell conditioned medium (ICCM) to unirradiated normal human keratinocyte cell line ( $\mathrm{HaCaT}$ ). Cell survival was investigated using the clonogenic assay, media transfer of $\mathrm{HaCat}$ cells exposed to low-LET $\gamma$ radiation $(0.5 \mathrm{~Gy})$ to by stander $\mathrm{HaCat}$ cells treated with oleic acid. The free radical scavenging property of oleic acid was performed by DCHFDA method and magnitude of lipid peroxidation was estimated with cisparineric acid as a probe using a fluorescence spectrometer. Mitochondrial membrane potential $(\Delta \psi \mathrm{m})$ was observed using $\mathrm{DiOC}_{6}$ as a sensitive probe visualized under a confocal microscope. Based on clonogenic assay, it was found that a significant reduction in clonogenic survival was seen in unirradiated cells exposed to low dose ICCM. Results have further shown that $\mathrm{HaCaT}$ cells pretreated with oleic acid before the medium transfer showed an increased percentage of clonogenic survival suggesting protection of cells against the ICCM factors. Free radical scavenging activity was also reported in cell membrane and mitochondria. A correlation between the antioxidant properties of $\mathrm{OA}$ and an increase in clonogenic survival was seen. These results showed that oleic acid was protecting normal cells from indirect low-dose $\gamma$ irradiated cells. It seems that ROS were involved in the mechanism of bystander effect which was significantly suppressed when OA was present.
\end{abstract}

Keywords: low dose radiation, bystander effect, oleic acid, radioprotection
Volume 9 Issue 3 - 2018

\author{
Abhay Puthli,' Reeta Tiwari, ${ }^{2}$ Kaushala Prasad \\ Mishra $^{3}$ \\ 'Department of Life Sciences, Medical Physics and Radiation \\ Sciences, University of Mumbai, India \\ ${ }^{2}$ Department of Life Sciences, Radiological Physics \& Advisory \\ Division, University of Mumbai, India \\ ${ }^{3}$ Department of Life Sciences, Nehru Gram Bharati University, \\ India
}
Correspondence: K.P.Mishra, President, Asian Association for Radiation Research (AARR), 2017-202I, Former Vice Chancellor,NGB University, Allahabad, Ex Head, RB\&HSD,Bhabha Atomic Res. Center,Mumbai, President, Foundation for Education and Research, India (FERI), Tel +91-8369845472/9838737787(WhatsApp)/9320466999, Fax+9|-22-250I 8I I4,Twitter @DrKPMishra Blog www. profkpmishraindia.blogspot.in,

Emailmishra_kaushala@rediffmail.com,mishradrkp@gmail.com

Received: May 15, 2018 | Published: June 20, 2018
Residence: 504, Neelyog Residency CHS Ltd, K-1,Pant Nagar, Ghatkopar(East), Mumbai 400075

Running title: Oleic acid protects normal bystander cells against indirect low dose radiation

Abbreviations: ICCM, irradiated cell conditioned medium; HaCaT, human keratinocyte cell line; HAECE. human aortic endothelial cells; TRAIL, tumor necrosis factor-related apoptosis inducing ligand; BARC, bhabha atomic research centre; MMP, mitochondrial membrane potential

\section{Introduction}

The olive tree, Olea europaea, is native to the Mediterranean basin and parts of Asia Minor. The fruit and compression extracted oil have a wide range of therapeutic and culinary applications. ${ }^{1}$ Olive oil also constitutes a major component of the "Mediterranean diet." The major active components of olive oil include oleic acid, phenolic compounds, and squalene. Studies on the oleic acid concentration present in food sources have shown that olive oil contains approximately $72 \%$, meats including beef and poultry contain (30-45)\%, while vegetable oils such as palm, peanut, soybean, and sunflower contain approximately in the range of (25-49)\% oleic acid. ${ }^{2}$ Interestingly, olive oil is unique with respect to its high oleic acid content which is a monounsaturated fatty acid whereas most other seed oils are composed primarily of polyunsaturated fatty acids, including the essential omega- 6 fatty acid. Compared to polyunsaturated fatty acids, oleic acid is monounsaturated, meaning it has one double bond, making it much less susceptible to oxidation and contributing to the antioxidant activity, high stability, and long shelf life. ${ }^{3}$ Keys et al., ${ }^{4}$ conducted the Seven Countries Study, which revealed the Mediterranean diet is linked to a reduced incidence of degenerative diseases, particularly coronary heart disease and cancers of the breast, skin, and colon, later studied and reviewed by him in 2004. Compared to diets of other countries, the Mediterranean diet has a relatively high fat content; however, as the diet is associated with a low incidence of cancer and coronary heart disease, despite the high fat intake, it has been suggested the type of fat is more important than the total amount consumed.

Several research investigators have performed in vitro and in vivo studies on the effect of oleic acid (OA) on cancer. Llor et al., ${ }^{6}$ conducted in vitro experiments on the effect of olive oil or isolated oleic acid on colorectal neoplasia studying protein expression of cyclooxygenase (COX-2) and B cell lymphoma 2(Bcl-2). COX-2 is believed to play an important role in colorectal cancer development, while Bcl-2 is an intracellular anti-apoptotic protein. They concluded that olive oil induced apoptosis and cell differentiation and downregulated the expression of COX-2. Apoptotic cell death specificity was seen in some cell lines like HT-29 cells but not Caco-2 cells. OA had no effect on the down-regulation of COX-2 and Bcl-2. Olive oil was found to have no effect on cell proliferation. The researchers concluded oleic acid played a minor role, if any; in colorectal chemoprotection and that other components of olive oil seemed likely to be involved in this protective process. ${ }^{6}$ Further research on chemosensitization was shown when OA was combined with paclitaxel, an enhancement of chemosensitivity was found. However, when OA was used concurrently with paclitaxel pretreatment of MDA-MB-231 cells with OA for $24 \mathrm{~h}$ prior to a $24 \mathrm{~h}$ paclitaxel exposure produced greater enhancement of paclitaxel sensitivity at high OA concentrations than the concurrent exposure to OA and paclitaxel. The OA-induced sensitization to paclitaxel was not due to the cytoxicity of the fatty acid itself. ${ }^{7}$ Chemoprotective studies on swiss mice showed no increase in the incidence of skin tumour 
formation when 10 Swiss mice were treated dermally once with $0.3 \mathrm{ml}$ of a $1.5 \%$ solution of 9,10-dimethylbenzanthracene in liquid paraffin and from three weeks later with undiluted oleic acid, twice weekly for 20 weeks. The treatment produced epidermal hyperplasia but no tumours. ${ }^{8}$ Interestingly, little or no evidence exists regarding the protective effect of oleic acid, from the indirect effects of low dose radiation and its induced bystander effects to normal cells.

It is well accepted that there may indeed be no such thing as a 'safe' level of radiation exposure. This concurs well with the observed non-targeted effects also called 'bystander' phenomenon. This is a mechanism whereby cells, not exposed to radiation, display radiation like damage if in the vicinity of irradiated cells. ${ }^{9}$ Transmission of the molecular signals involved in bystander effects by intercellular communication through gap junctions ${ }^{10}$ or by release of factors into the suspension culture medium has been reported. ${ }^{11}$ and it has been suggested that irradiated cells secrete cytokines or other factors that induce an increased intracellular level of reactive oxygen species in unirradiated cells. ${ }^{12}$ The transmitted factors are diverse and may depend on the cell type or its physiological state, mediated by reactive oxygen species. Interleukin 8 , transforming growth factor beta (TGF $\beta$ ), the soluble death ligands Fas, tumor necrosis factorrelated apoptosis inducing ligand (TRAIL), and tumor necrosis factor $\alpha(\mathrm{TNF} \alpha)$ secreted by $\gamma$ irradiated cancer cells have been proposed as bystander messengers. ${ }^{13}$ Recently, in some studies, oleic acid has shown to be beneficial against TNF- $\alpha$-induced oxidative stress in cardiomyocytes. ${ }^{14}$ Previous study by Harvey KA et al., ${ }^{15}$ has shown that $\mathrm{OA}(25 \mu \mathrm{M})$ protected and reduced inflammatory effects induced by steric acid in human aortic endothelial cells (HAECE). In their paper, $\mathrm{OA}$ as low as $5 \mu \mathrm{M}$ also inhibited the steric acid-induced increase in intercellular adhesion molecule-1 (ICAM-1) expression. Stearic acid-induced phosphorylation of nuclear factor-kappa B (NF- $\kappa B)$, a transcriptional regulator of ICAM-1, was also reduced by oleic acid. Since there was a link between Oleic acid being protective against inflammatory mediators, we thought it interesting to investigate its protective role against the inflammatory mediators present in the low dose irradiated bystander medium (ICCM).

Present work investigated the effects of low dose radiation induced bystander effects on normal human keratinocytes, HaCat cells in vitro. In the panorama of the numerous established cell lines, the human keratinocyte line $\mathrm{HaCaT}$ has a very interesting feature, having a close similarity in functional competence to normal keratinocytes. This cell line has been used in many studies as a paradigm for epidermal cells and, therefore, we selected $\mathrm{HaCaT}$ as a cell model for investigating the protective effects of oleic acid against these bystander factors that are released during low dose exposure $(<0.5 \mathrm{~Gy})$ to skin and hair. The aim of the present study was to develop protocol to protect normal cells against the harmful effects of the bystander factor(s). These factors are known to cause genomic instability and cancer progression in vivo and in vitro. Radioprotective properties of OA were investigated as a candidate since it is cheap, easily available and nontoxic in the physiological range having anticancer properties. The use of dietary, nutraceutical supplementation or topical skin formulation of OA could protect the skin which is the first organ to receive low doses of radiation exposure during an accidental incident.

\section{Materials and methods}

\section{Chemicals and reagents}

Dulbecco's Modified Eagle's Medium(DMEM), fetal bovine serum
(FBS), penicillin, streptomycin and trypsin EDTA were purchased from Gibco Co., USA, carbol fushin, 3, 3'-dihexyloxacarbocyanine iodide $\left(\mathrm{DiOC}_{6}\right)$, Cisparineric acid $(\mathrm{cPnA})$, were purchased from Sigma (St. Louis, MO, USA).

\section{Culture conditions}

The HaCaT cells were grown in DMEM medium containing $10 \% \mathrm{FCS}$ (fetal calf serum) supplemented with glutamine $(2 \mathrm{mmol} / \mathrm{l})$, Hepes $(20 \mathrm{mmol} / \mathrm{l})$, streptomycin $(10 \mathrm{mg} / \mathrm{ml})$, penicillin $(10,000$ international units $/ \mathrm{ml})$ and sodium bicarbonate $(24 \mathrm{mmol} / \mathrm{l})$. Cells were grown in $25 \mathrm{ml}$ flasks containing $(0.1-1) \times 106$ cells $/ \mathrm{ml}$. The cells were kept in a humidified atmosphere at $37^{\circ} \mathrm{C}$ containing $5 \% \mathrm{CO}_{2}$.

\section{Irradiation}

Exponentially growing donor $\mathrm{HaCaT}$ cells in $\mathrm{T}-25$ flasks were sealed with parafilm wrap and covered in aluminum foil for transport to be irradiated. Radiation dose was given $6 \mathrm{~h}$ after plating using a cobalt-60 teletherapy source (Nordion Teletherapy machine installed at Bhabha Atomic Research Centre (BARC), India at a dose rate: $1.95 \mathrm{~Gy} / \mathrm{min}$.). To confirm the exact dose given, TLD badges were used. Flasks were returned to the incubator immediately after irradiation.

\section{Clonogenic assay technique}

The clonogenic assay technique was performed as mentioned earlier by Ryan LA et al. ${ }^{16}$ Briefly, cells were detached from flasks by incubating in a $0.25 \%$ trypsin-EDTA solution. After detachment, fresh medium was added and syringed gently to produce a single cell suspension. To determine the number of viable cells an aliquot of the cell suspension was mixed with trypan blue dye and counted using a hemocytometer. Appropriate cell numbers were plated for survival analysis using the Clonogenic assay technique by Puck et al. ${ }^{17}$ The number of cells plated was selected to be in the range that ensured linear relationship between the number of cells plated and the number of colonies returned. Cell cultures were incubated for 12 days at $37^{\circ} \mathrm{C}$ in 5\% $\mathrm{CO}_{2}$ in air and $95 \%$ relative humidity. The cells were then stained with $15 \%$ Carbol Fuschin and colonies exceeding 50cells were scored as representing surviving cells. To compare treatments, percentage survival was calculated using the formula: Percent Survival=100x[PE of the treatment/PE of controls]. PE stands for plating efficiency determined by number of colonies counted/number of colonies plated.

\section{Bystander protocol}

The cell suspension after dilution was counted using a hemocytometer. Appropriate cell numbers were plated as per the Puck and Marcus technique in $5 \mathrm{ml}$ medium in $25 \mathrm{~cm}^{2}$ NUNC flasks. There were three groups of flasks: those designated for control or un-irradiated, by stander donor flasks and by stander recipient flasks. Bystander donor flasks were heavily seeded with cells $(0.3 \times 106$ cells/ flask) and exposed to $0.5 \mathrm{~Gy} \gamma$ radiation and the medium harvested after $6 \mathrm{hrs}$ and passed through a microfilter to remove any cells. This cell free medium is referred to as the Irradiated Cell Condition Medium (ICCM). Bystander recipient flasks were set up with the normal cloning number (approximately 300cells) and received no treatment except the bystander medium (ICCM) from the bystander donor flasks. Oleic acid at $(10 \mu \mathrm{M})$ was added alone and also to the bystander recipient flasks that were seeded with approximately 300cells/flask for $2 \mathrm{hr}$ in serum free media. After $2 \mathrm{hr}$ with OA treatment bystander recipient flasks were washed with PBS and ICCM was added to them. 
Each of the four groups was designated as: untreated control, ICCM control, oleic acid only and oleic acid treated with ICCM were set in triplicates. In all the experiments, autologous medium transfer took place meaning irradiated normal keratinocyte cells donor ICCM was transferred to recipient normal keratinocyte cells.

\section{Estimation of intracellular ROS}

The generation of intracellular ROS was measured using DCHFDA (2,7-dichlorodihydrofluorescein diacetate), a florescence probe as described previously. ${ }^{18}$ Briefly, $\mathrm{HaCaT}(2 \times 105 \mathrm{cells} / \mathrm{ml})$ were incubated with OA with respective controls in phosphate buffered saline (PBS) for $1 \mathrm{~h}$ at $37^{\circ} \mathrm{C}$ followed by labeling with the fluorescence probe after irradiation of the samples. Aliquots of $200 \mu 1$ obtained after different treatments were diluted to $3 \mathrm{ml}$ with $\mathrm{PBS}$ followed by measurement of fluorescence intensity ( $\lambda=490$ and $520 \mathrm{~nm}$ ) in quartz cuvette using Fluorescence Spectrometer (LS50B, Perkin Elmer. USA).

\section{Lipid peroxidation in $\mathrm{HaCaT}$ cells}

HaCaT cells $(2 \times 105 / \mathrm{ml})$ were loaded with $\mathrm{cPnA}(10 \mu \mathrm{M})$ for $30 \mathrm{~min}$. at room temperature in dark, later treated with different treatment groups and incubated at $37^{\circ} \mathrm{C}$ in a humidified atmosphere of $5 \% \mathrm{CO}_{2}$ for $2 \mathrm{~h}$. After $2 \mathrm{~h}$, the cells were washed with PBS twice, and loss of flourescence was monitored for $1 \mathrm{~min}$ at $\lambda \mathrm{ex} / \lambda \mathrm{em}=318 / 420 \mathrm{~nm}$ in quartz cuvette using Fluorescence Spectrometer (LS50B, Perkin Elmer. USA). Loss of fluorescence from cis-parinaric acid (cPnA) was taken as indicator of lipid peroxidation.

\section{Mitochondrial membrane potential $(\Delta \psi \mathrm{m})$}

Cells at concentration of $1 \times 105 / \mathrm{ml}$ in PBS containing $1 \%$ BSA was incubated with $40 \mathrm{~nm}$ of $\mathrm{DiOC}_{6}$ probe for $20 \mathrm{~min}$ in the dark at $37^{\circ} \mathrm{C}$. $20 \mathrm{~min}$. incubation allows the probe to enter the cytoplasm and mitochondrial matrix. Later, ICCM, OA and combination of both were added to the samples and kept in dark for $2 \mathrm{~h}$ in the $\mathrm{CO}_{2}$ incubator. After treatments, the samples were washed two times in PBS so as to remove all traces of the probe and loss of fluorescence was observed using a confocal microscope at $\lambda \mathrm{ex} / \lambda \mathrm{em}=485 / 525 \mathrm{~nm}$

\section{Statistical analysis}

The clonogenic survival data are presented as a mean $\pm \mathrm{SEM}$, where each treatment had $\mathrm{N}=3$ recipient flasks. Similarly, measurement of ROS and lipid peroxidation experiments were repeated twice and data are presented as means \pm SEM. The statistical significance of the difference between treated and control groups were done by Student's t-test using graph pad software. In all statistical analysis, $p<0.05$ were accepted as significant.

\section{Results}

\section{Cell survival after treatment with OA, ICCM and combined}

Figure 1 shows the clonogenic survival of different treatment groups either alone or in combination with OA. Results have shown that a significant decrease in the survival of clones was observed in the ICCM treated group, resulting in $85 \%(\mathrm{p}<0.05)$ of clonal survival. An insignificant decrease in clonogenic survival was found in OA treated group as survival was comparable to the control group. However, the combined effect of OA and ICCM showed a non-significant decrease in survival to $98.8 \%(\mathrm{~ns})$. Results have shown that inclusion of OA increased the survival of ICCM induced toxicity to normal cells by almost $28 \%(\mathrm{p}<0.05)$

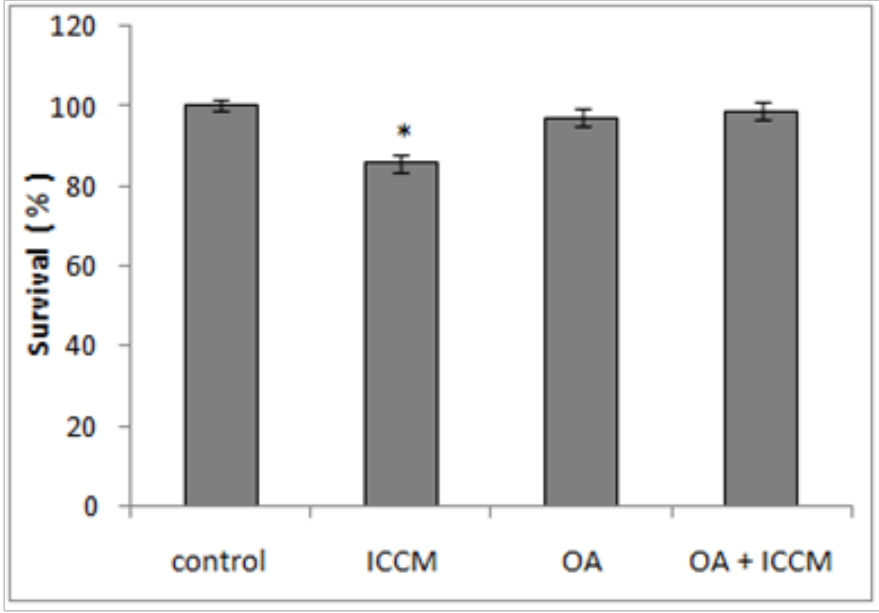

Figure I Survival of $\mathrm{HaCaT}$ cell line showing control, cells exposed to irradiated cell conditioned medium (ICCM), I0 $\mu$ M Oleic acid (OA) and $10 \mu \mathrm{M}$ Oleic acid pretreated and exposed to ICCM media (OA+ICM). Values are $\pm S E M$ for $n=3$ from one experiment.

$*(\mathrm{P} \leq 0.05)$ was considered statistically significant.

\section{Generation of ROS after treatment with OA and ICCM}

Figure 2 shows the generation of reactive oxygen species of the various treatment groups in normal cells. Results have shown that ICCM induced an increase in ROS generation as measured by DCHFDA fluorescent probe; it was a 2 -fold increase as compared to the control group which was highly significant $(\mathrm{p}<0.001)$. Oleic acid treated cells did not show any increase in ROS generation. It was interesting to observe that, pretreatment of OA to the cells treated with ICCM resulted in a significant decrease of ROS production, when compared to the ICCM control group $(\mathrm{p}<0.01)$.

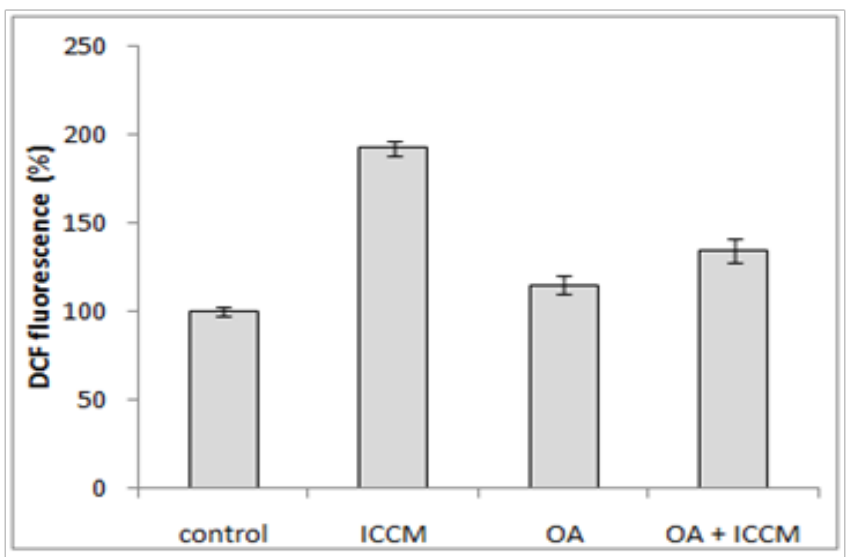

Figure 2 Measurement of reactive oxygen species (ROS) using DCHFDA(2,7dichlorodihydrofluorescein diacetate), as a florescence probe.

\section{Estimation of lipid peroxidation}

Figure 3 gives the decrease in fluorescence of the $\mathrm{DiOC}_{6}$ probe as a sensitive indicator of lipid peroxidation. Here, we observed a significant decrease in the fluorescence, when cells were treated with only ICCM $(\mathrm{p}<0.01)$. However, an insignificant or no decrease was observed in either of the OA and combined OA with ICCM treated groups. Mitochondrial membrane potential (MMP) 


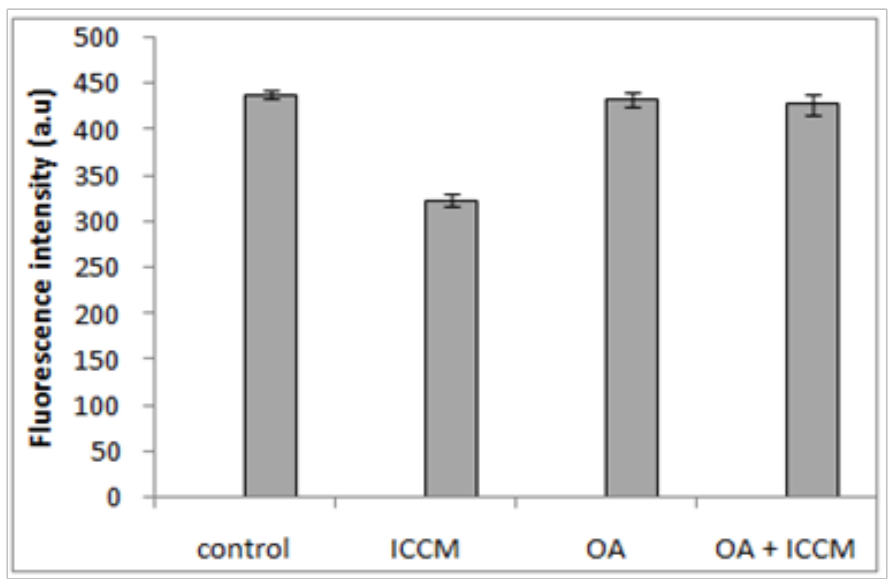

Figure 3 Loss of fluorescence measurement in $\mathrm{HaCaT}$ cells using cis-parinaric acid (cPnA) as a sensitive indicator of lipid peroxidation.

Figure 4 shows images of the fluorescence intensity corresponding to mitochondrial membrane potential in the different treated groups as a visual comparison, visualized under the confocal microscope. We observed that when cells were treated with ICCM there was a significant decrease in the fluorescent intensity of the $\mathrm{DiOC}_{6}$ dye as compared to the control which glowed at a much brighter intensity. It was also observed that the OA treated group and the combined treated group showed similar fluorescence intensities as the control.

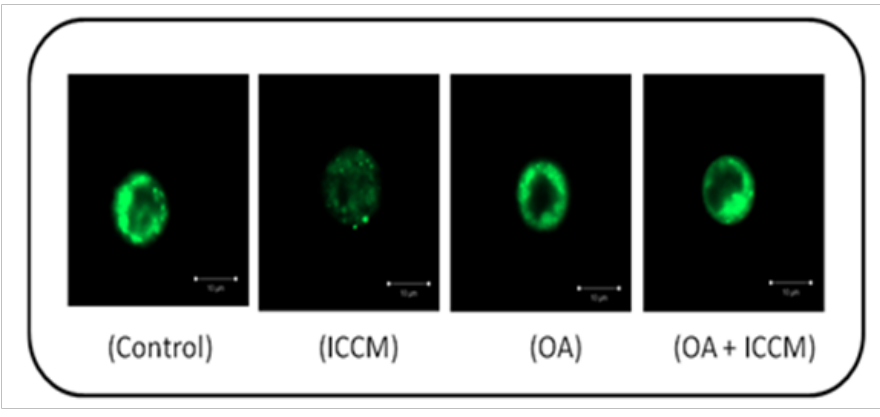

Figure 4 Images show loss of mitochondrial membrane potential (MMP) captured under confocal microscopy.

\section{Discussion}

The bystander effect is a well-established biological response to low dose radiation in-vio and in-vitro., ${ }^{91,20-22}$ Results from our experiments in Figure 1 have shown that there was a significant decrease $(p \leq 0.05)$ in survival after ICCM was transferred onto unexposed cells of the same cell line. Our results agree with previous published studies using the same cell line and radiation dose when clonogenic survival was used as the biological end point. ${ }^{11}$ The concentration of oleic acid in healthy human plasma ranges from approximately $50 \mu \mathrm{M}^{23}$ to approximately $200 \mu \mathrm{M}^{24}$ due to several factors such as age, race, physical activity, diet and method of quantification. Due to the high variable nature of free oleic acid plasma levels we decided to choose a definitive concentration present in all types of humans and investigated its cytotoxicity on our cell line by including $10 \mu \mathrm{M}$ OA in the medium which did not reduce the clonogenic survival. These results were similar to Martins T et al, (2006) whose toxicity study of OA on a macrophage cell line (J774) showed that OA was toxic only at high concentrations $(>200 \mu \mathrm{M})$. Since physiological concentrations of OA being nontoxic we sought to investigate its protective effect against the bystander factors. In our study, we treated cells with $10 \mu \mathrm{M}$ of OA prior to the ICCM and noticed a significant increase in clonogenic survival $(\mathrm{p}<0.05)$. Similarly, OA $(50 \mu \mathrm{M})$ incorporation protected cultured hamster fibroblasts from 95\% oxygen-induced cytotoxicity increasing its cell survival. ${ }^{25}$ To our knowledge, this is the first study indicating that OA can protect normal cells, from the toxic effects induced by low dose radiation bystander factors. Work done by Pandey BN et al., ${ }^{12}$ have demonstrated an increase in intracellular reactive oxygen species (ROSs) and alterations in mitochondrial membrane potential (MMP) in his medium transfer experiments. Since ROS and MMP are implicated in the bystander effect, we decided to investigate if OA could mitigate ROS and protect normal cells. Our results in Figure 2 have shown a significant 2 -fold increase $(p<0.001)$ in intracellular generation of ROS to cells exposed to ICCM, confirming Panday BN et al work. On the other hand, OA alone did not generate any significant levels of ROS, which agrees with the results reported by Smith GI et al., ${ }^{26}$ showing that a high intake of fatty acids had little effect on markers of inflammation and oxidative stress in humans. However, interestingly OA pretreatment to the ICCM resulted in inhibiting ROS generation and decreased the fluorescence intensity significantly $(\mathrm{p}<0.01)$ when compared to ICCM exposed cells. Cell membranes are known to be the target sites for free radical damage and, therefore, we attempted to elucidate if oxidative stress caused lipid peroxidation in the ICCM exposed cells. We used DiOC $_{6}$ probe as a sensitive indicator of lipid peroxidation and data from Figure 4 have shown a significant decrease in the fluorescence, when cells were treated with only ICCM $(p<0.01)$ indicating that lipid peroxidation occurred in bystander cells. This was in accordance with the results of Chakraborty A et al. ${ }^{27}$ who showed that oxidative stress in the bystander population was reduced by the presence of the membrane antioxidant, vitamin $\mathrm{E}$, in the targeted cells, suggesting that lipid peroxidation may play a key role in mediating these bystander effects. Confirming Chakraborty's suggestions, we replaced Vitamin $\mathrm{E}$ with $\mathrm{OA}$ and our results in Figure 3 have shown that, antioxidant properties of OA prevented oxidative stress induced lipid peroxidation in bystander cells exposed to ICCM. Confirming previous electron paramagnetic resonance (EPR) spectrometry studies regarding protective action of oleic acid against iron-induced oxidant stress, the incubation of endothelial cells with $5-20 \mu \mathrm{M}$ oleic acid decreased oxidative stress. Likewise, when U937 monocytes treated with docosahexaenoic acid (DHA) were exposed subsequently to $20 \mu \mathrm{M}$ oleic acid showed a $35-45 \%$ decrease in radical adduct formation also occurred, whereas incubation with polyunsaturated fatty acids such as linoleic acid increased lipid peroxidation..$^{28}$ Mitochondria are the major source of ROS, since it has been estimated that $1 \pm 2 \%$ of consumed oxygen is converted into the superoxide anion $\left(\mathrm{O}_{2}^{-}\right)$, mainly generated at complexes I and III of the electron transport chain. $\mathrm{O}_{2}^{-}$ is converted into $\mathrm{H}_{2} \mathrm{O}_{2}$ by the mitochondrial SOD. ${ }^{29}$ Pandey BN and his colleagues (2011) have investigated the effect of ICCM, obtained from human promyelocytic leukemic cells (HL-60) $\gamma$-irradiated either with low-dose $(5 \mathrm{cGy})$ or high-dose radiation (1Gy) on unirradiated HL-60 and normal human blood lymphocytes showing alterations in mitochondrial membrane potential. Likewise, similar observations were seen in our confocal results Fugure 4 showing a decrease or loss in fluorescence intensity in ICCM treated cells indicating mitochondrial damage. Interestingly, there was no change in the fluorescence intensity of OA pretreated with ICCM which was as bright as the control group flourescence suggesting that OA was 
responsible for the prevention and preservation of mitochondria from oxidative stress. Similar work done by Duval C et al., ${ }^{30}$ and coworkers have shown that OA could counterbalance mitochondrial ROS generation elicited by antimycin $\mathrm{A},{ }^{31}$ in human ECV-304 cells, and that this antioxidant effect resulted from an epidermal growth factor receptor (EGFR) mediated glutathione peroxidase enzyme activation and subsequent ROS degradation. Our results have shown a correlation between antioxidant activities of OA and clonogenic survival that resulted in an increase in the number of colonies due to degradation of intracellular ROS generation via ICCM.

\section{Conclusion}

Present results have allowed us to conclude that oleic acid protected normal cells against oxidative stress mediated cytotoxicity from low dose $\gamma$ radiation induced bystander factors in normal human keratinocytes, HaCat. It seems that ROS were involved in the mechanism of bystander effect which was significantly suppressed when OA was present. Role of mitochondrial membrane potential was found in the mechanism of bystander effect. Our results suggest that oleic acid supplementation in diet or as neutraceuticals may help protect normal cells and tissues of cancer patients undergoing radiotherapy, victims of radiation accidents and astronauts receiving low dose radiation in space.

\section{Acknowledgements}

This research was made possible through the grant support provided by the Canada Indo Shastri foundation. The authors thank, Carmel Mothersill, Collin Seymour and Harleen Singh. Medical Physics and Radiation Sciences, McMaster University for their help during experiments. We further thank Dr. Balvinder Sapra and Dr Sreedevi Balkrishnan, RPAD, BARC for their encouragement.

\section{Decliration of interest}

The authors declare that there are no conflicts of interest. The authors alone are responsible for the content and writing of this paper.

\section{References}

1. Wahrburg U, Kratz M, Cullen P. Mediterranean diet, olive oil and health. Eur J Lipid Sci Technol. 2002;104(9-100:698-705.

2. Newmark HL. Squalene, olive oil, and cancer risk: a review and hypothesis. Cancer n Epidemiol BiomarkersPrev. 1997;6(12):11011103.

3. Owen RW, Mier W, Giacosa A, Hull WE, et al. Phenolic compounds and squalene in olive oils: the concentration and antioxidant potential of total phenols, simple phenols, secoiridoids, lignans and squalene. Food Chem Toxicol. 2000;38(8):647-659.

4. Keys A, Menotti A, Karvonen MJ, et al. The diet and 15-year death rate in the Seven countries study. AmJ Epidemiol. 1986;124(6):903-915.

5. Owen RW, Haubner R, Würtele G, et al. Olives and olive oil in cancer prevention. Eur J Cancer Prev. 2004;13(4):319-326.

6. Llor X, Pons E, Roca A, et al. The effects of fish oil, olive oil, oleic acid and linoleic acid on colorectal neoplastic processes. Clin Nutr. 2003;22(1):71-79.

7. Menéndez JA, del Mar Barbacid M, Montero S, et al. Effects of gammalinolenic acid and oleic acid on paclitaxel cytotoxicity in human breast cancer cells. Eur J Cancer. 2001;37(3):402-413.
8. Shubik P. Studies on the promoting phase in the stages of carcinogenesis in mice, rats,rabbits, and guinea pigs. Cancer Res. 1950;10(1):13-17.

9. Mothersill C, Seymour CB. Medium from irradiated human epithelial cells but not human fibroblasts reduces the lonogenic survivalof unirradiated cells. Int J Radiat. Biol. 1997;71(4):421-427.

10. Azzam EI, Toledo SM, Little JB. Stress signaling from irradiated to non irradiated cells. Curr Cancer Drug Targets. 2004;4(1):53-64.

11. Mothersill C, Seymour CB. Cell-cell contact during gamma irradiation is not required to induce a bystander effect in normal human keratinocytes. Radiat Res. 1998;149(3):256-262.

12. Pandey BN, Kumar A, Ali M, et al. Bystander effect of conditioned medium from low and high doses of $\gamma$-irradiated human leukemic cells on normal lymphocytes and cancer cells. J Environ Pathol Toxicol Oncol. 2011;30(4):333-340.

13. Desai S, Kumar A, Laskarb S, et al. Cytokine profile of conditioned medium from human tumor cell lines after acute and fractionated doses of gamma radiation and its effect on survival of bystander tumor cells. Cytokine. 2013;61(1):54-62.

14. Al-Shudiefat AA, Sharma AK, Bagchi AK, et al. Oleic acid mitigates TNF- $\alpha$-induced oxidative stress in rat cardiomyocytes. Mol Cell Bioche. 2013;372(1-2):75-82.

15. Harvey KA, Walker CL, Xu Z, et al. Oleic acid inhibits stearic acidinduced inhibition of cell growth and pro-inflammatory responses in human aortic endothelial cells. J Lipid Res. 2010;51(12):3470-3480.

16. Ryan LA, Seymour CB, O'Neill-Mehlenbacher A, et al. Radiationinduced adaptive response in fish cell lines. $J$ Environ Radioact. 2008;99(4):739-747.

17. Puck TT, Markus PI. Action of x-rays on mammalian cells. $J$ Exp Med. 1956;103(5):653-66.

18. Puthli A, Tiwari R, Mishra KP. Biochanin A enhances the radiotoxicity in colon tumor cells invitro. $J$ Environ Pathol Toxicol Oncol. 2013;32(3):189-203.

19. Mothersill C, Rea D, Wright EG, et al. Individual variation in the production of a 'bystander signal' following irradiation of primary cultures of normal human urothelium. Carcinogenesis. 2001;22(9):14651471.

20. Mothersill C, Seymour CB, Joiner MC. Relationship between radiationInduced low-dose hypersensitivity and the bystander effect. Radiat Res. 2002;157(5):526-532.

21. Mothersill C, Bucking C, Smith RW, et al. Communication of radiationinduced stress or bystander signals between fish in vivo. Environ $\mathrm{Sci}$ Technol. 2006;40(21):6859-6864.

22. Mothersill C, Smith RW, Agnihotri N, et al. Characterization of a radiation-induced stress response communicated in vivo between zebrafish. Environ Sci Technol. 2007;41(9):3382-3387.

23. Teubert A, Thome J, Büttner A, et al. Elevated oleic acid serum concentrations in patients suffering from alcohol dependence. $J \mathrm{Mol}$ Psychiatry. 2013;1(1):13

24. Fraser DA, Thoen J, Rustan AC, et al. Changes in plasma free fatty acid concentrations in rheumatoid arthritis patients during fasting and their effects upon T-lymphocyte proliferation. Rheumatology (Oxford). 1999;38(10):948-952.

25. Kinter M, Spitz R, Roberts J. Oleic acid incorporationprotects cultured hamster fibroblastfrom oxygen induced cytotoxicity. $J$ Nutr. 1996;126(12):2952-2959. 
26. Smith GI, Atherton P, Reeds DN, et al. Dietary omega-3 fatty acid supplementation increases the rate of muscle protein synthesis in older adults: a randomized controlled trial. Am J Clin Nutr. 2011;93(2):402412 .

27. Chakraborty A, Held KD, Prise KM, et al. Bystander effects induced by diffusing mediators after photodynamic stress. Radiat Res. 2009;172(1):74-81.

28. North JA, Kiminyo KP, Buettner GR, et al. Polyunsaturated fatty acids increase lipid radical formation induced by oxidant stress in endothelial cells. J Lipid Res. 1994;35(10):1773-1785.
29. Loschen G, Azzi A, Richter C, et al. Superoxide radicals as precursors of mitochondrial hydrogen peroxide. FEBS Lett. 1974;42(1):68-72.

30. Duval C, Augé N, Frisach MF, et al. Mitochondrialoxidativestress is modulated by oleicacidvia an epidermal growth factor receptordependent activation of glutathione peroxidase. Biochem J. 2002;367(pt 3):889-894.

31. Martins de Lima T, Cury-Boaventura MF, Giannocco G, et al. Comparative toxicity of fatty acids on a macrophage cell line (J774). Clin Sci. 2006;111(5):307-317. 